\title{
The junkyard dogs find their teeth: addressing the crisis of admitted patients in Canadian emergency departments
}

\author{
Riyad B. Abu-Laban, MD, MHSc
}

$\mathrm{I}_{\mathrm{p}}^{\mathrm{n}}$ n the Fall of 2005, after years of effort by emergency physicians at Vancouver General Hospital (VGH) to address the crisis of admitted patients in our ED, the Senior Medical Director at VGH introduced the topic at a medical staff meeting by saying we were "behaving like junkyard dogs." On Apr. 3, 2006, the junkyard dogs bit back, when a majority of VGH emergency physicians began giving selected patients a one-page statement declaring "non confidence in the ability of the VGH Emergency Department to provide safe, timely and appropriate emergency medical care." The statement explained that "despite initiatives to improve the situation, the approach to hospital overcrowding continues to involve an excessive, inappropriate, and unsafe use of the ED to house admitted patients." It went on to outline a recommendation to temporarily address this problem using existing hospital resources, by sharing the excess workload equally throughout the hospital rather than placing a disproportionate share of the burden in the ED. This was a recommendation we had been unsuccessfully lobbying administration to implement for years. The statement ended by informing patients we were "outraged with the deplorable situation" they were likely to find themselves in, and urged them to help us in our efforts to improve things by contacting the Chief Operating Officer and Chief Executive Officer of the hospital and the Provincial Minister of Health.

The decision to declare non-confidence in our ED was not taken lightly, nor was it easily or unanimously reached.
It arose after years of seeing our patient care deteriorate despite huge efforts and advocacy on our part. Of course this situation at VGH is not unique. The identical problem exists to varying degrees in most major Canadian EDs, and has caused many emergency groups and ED leaders to engage in increasingly acrimonious interactions. However, a public declaration of non-confidence at a flagship teaching hospital, and the magnitude of events that followed, are to my knowledge unprecedented in the history of Canadian emergency medicine. Reflecting on these events, and on the systemic issues that led to the VGH action, may provide insight into how to address the crisis of admitted patients in Canadian EDs.

As awareness of the VGH action spread, reaction was swift. Within 48 hours the government's official opposition, quoting directly from our patient statement in the provincial legislature, launched a verbal assault on the BC government; an event that led the evening TV news. Meanwhile, VGH administration took particular issue with our use of the word "unsafe," and reassuringly declared to a skeptical public and media that if the ED were indeed unsafe they would have closed it. Our administration also demanded we "cease and desist" the action, which they deemed to be "unprofessional." Over the next week circulation of the statement was suspended while members of our group communicated with the BC Medical Association, the BC College of Physicians and Surgeons, the Canadian Medical Protective Association, the Canadian 
Association of Emergency Physicians (CAEP), and our own legal council. Although no formal judgment of our action was ever made, on April 12th, the Registrar of the BC College of Physicians and Surgeons was quoted in The Vancouver Sun as saying "Regardless of the validity of the doctors' concerns, there is no need to further aggravate patients by telling them their care may be compromised. That

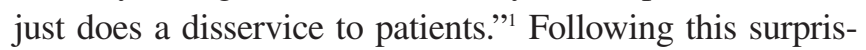
ing statement, which contrasted with information the College conveyed in a prior meeting with our group, the media flurry abruptly ended, as did any thoughts of resuming circulation of the statement as originally worded.

In the days that followed, it became clear that the VGH ED nurses, the public, and media pundits all understood and supported what the emergency physicians had done. Fifty-six VGH ED nurses signed an unsolicited letter to administration in support of the action that was also noteworthy in stating "we believe the department is unsafe." A citizen's letter to the editor of The Vancouver Sun stated "One can only conclude that this is an act of desperation from a group whose concerns are being ignored. Why else would this group of professionals stick their necks out on behalf of emergency patient care by stepping into the political hot seat?"2 The letter went on to criticize the College, as did a Vancouver Sun columnist, who wrote "Oh yes, let's shut down this controversy and allow the public to find out about this holy mess only when they actually visit VGH's emerg."” Meanwhile, groups of emergency physicians from the Royal Columbian Hospital and Lions Gate Hospital, both in the greater Vancouver area, came forward to the media with unsolicited letters of support. On April 25th an articulate and experienced emergency physician from the Royal Columbian Hospital hit the front page of The Vancouver Sun, fists clenched in frustration in the accompanying photo, and referred to a leaked internal report saying “I can't do my job. People have been dying on ambulance stretchers in our emergency room, and here is a document that actually confirms it."' The explosive headline of The Vancouver Sun that day read "Overcrowded ER Killing Patients at Royal Columbian, Report Says." Over the next 4 days, as other doctors and information flooded forward, the story remained glued to the front page of The Vancouver Sun with the following headlines: "Health minister rejects MDs' ER Warning As Alarmist"; "We Warned of ER Deaths: Doctors Complaining of Sub-
Par Care For 3 Years, Report Shows"; "MDs Reveal ER Horror Stories"; and "15 of Region's ERs Not Up To National Standards."

On April 30th, in the face of massive media attention and opposition pressure, the BC Health Minister finally acknowledged a problem indeed existed and, following a hastily-called provincial meeting of health care providers and administrators, announced the release of $\$ 7$ million to fund a campaign encouragingly named Action Now. The stated purpose of the campaign was to "apply innovation, think outside the box, and break through routine bureaucracy to enable immediate action" to address the provincial ED situation. Unfortunately, Action Now has to date been inappropriately focused on the exploration of internal ED process improvements, rather than on issues surrounding admitted patients such as service accountability, common safety standards, and workload sharing. After a summer lull, the VGH ED looks increasingly like it did a year ago: gridlocked with admitted patients while the front hallway is full of new patients receiving undignified, unsafe care by frustrated EPs, nurses and paramedics. And once again my group is seriously considering taking strong action.

As I look back on these tumultuous events in BC, and on the evolution of our specialty since I worked my first ED shift over 20 years ago, many questions come to mind. Why did things come to this? How could it be that a group of emergency physicians would feel compelled to publicly declare non-confidence in their ED? Why was support from colleagues in other specialties minimal and predominately provided only in hushed hallway conversations? Why was VGH administration more focused on suppressing our message than on acknowledging its accuracy and genuinely seeking a solution? Why wasn't our action alone enough to stimulate an appropriate response from the government, rather than initial claims by the Health Minister that we were "alarmist"? Why was it only after other EDs and individual emergency physicians bravely came forward with the same message that the government finally reacted positively? In fact, why wasn't Action Now initiated years ago, in response to the compelling reports from emergency medicine administrative leaders in BC and other efforts and actions by various emergency groups? And finally, looking beyond $\mathrm{BC}$, how is it possible that despite years of national effort, Canada's emergency physi- 
cians have still failed to address the greatest barrier to safe, timely and appropriate delivery emergency care: the boarding of admitted patients for hours and often days, and its lethal clogging effect on ED function?

Of course the answers to such questions are difficult and multifactorial, but in reflecting upon them I believe we must acknowledge a painful truth - we in emergency medicine bear a significant share of the responsibility for the situation we currently face. Why? Because we have not advanced and rigorously defended standards regarding the parameters of ED care and this, combined with our reactive problem-solving approach to the endless encroachment of admitted patients on ED space and human resources, has enabled the situation to develop and worsen. It is an accepted fact that our current lack of hospital capacity is a result of complex systemic problems without a simple solution. However, both individually and organizationally, we have bought into and perpetuate the myth that the causes and solutions to ED overcrowding are similarly complex. In fact the overwhelmingly greatest single cause of ED overcrowding is our inappropriate and unsafe approach to dealing with a lack of hospital capacity (not the lack of capacity itself). As such, there is an obvious immediate solution to ED overcrowding that could be implemented while the long-term problems are being addressed: change the approach to dealing with a lack of hospital capacity so it is more appropriate and safe. This solution doesn't require any additional funds; it simply requires vision and political will on the part of senior health administration.

The best-known intelligently designed system in health care to deal with demand exceeding capacity is a disaster plan. Not surprisingly, the approach in such plans is to spread the workload evenly throughout the institution, thus doing the most good, and the least harm, with whatever resources are available. This is the essence of the "overcapacity protocol," made famous after its first successful implementation years ago at Stony Brook University Hospital (now Stony Brook University Medical Center) in New York. Such protocols rapidly send admitted patients to hospital wards regardless of whether ideal staffing or even a physical bed exists. This logical immediate solution is not simply a temporizing measure, it's a safer and better way to deal with capacity shortfalls whenever they occur. In addition, overcapacity protocols have been shown to increase efficiency in ward throughput. They also encourage broader physician, nurse and administrative involvement in the quest for solutions to overcrowding.

As a result of both prior and ongoing efforts by emergency medicine administrative leaders in $\mathrm{BC}$, the events of this Spring, and the subsequent Action Now process, the BC Ministry of Health verbally endorsed the concept of overcapacity protocols. It is encouraging that such protocols have since been implemented by some health authorities, and in a few hospitals they have profoundly improved the ED situation. However, in most locations, including $\mathrm{VGH}$, the protocols have not been appropriately designed or faithfully implemented in a manner that can truly address the problem.

In an ACEP News editorial this August, the architect of the Stony Brook overcapacity protocol, Dr. Peter Viccellio, was blunt in his assessment of the cause of the current ED situation. He wrote: "The first step to solving this overcrowding problem is to understand fully and correct the origin of the problem. The problem is us. We are, in a word, pushovers." ${ }^{5}$ Changing our current paradigm of individual and organizational behaviour to better protect the interests of ED patients will require a collective re-evaluation of our strategies. CAEP, as our national organization, has an essential role to play in this regard and, in my view, should focus significant energy and resources on the development and dissemination of position statements and standards relating to our domain of expertise: the parameters of the practise of emergency medicine and ED function. These should include the appropriate total throughput time for ED patients, the role of EDs in the care of admitted patients, the appropriate time from an admission request to a patient exiting the $\mathrm{ED}$, and the role of overcapacity protocols. Our lack of national standards beyond CTAS $^{6}$ (Canadian Emergency Department Triage and Acuity Scale), and our predominately narrow focus on the long-term capacity solution of increasing bed numbers, are major impediments to forcefully dealing with the immediate crisis ED patients now face. Our provincial organizations also have a role to play in this regard, and some have recently done so. In June, the BC Medical Association Section of Emergency Medicine released a document titled "Position Statement on Emergency Department Overcrowding and Proposed Short Term Solution." This called for a 2-hour provincial standard from the time a bed 
is requested for an admission until the patient exits the ED (regardless of hospital capacity). The front page headline of The Vancouver Sun the next day read: "MDs Propose Solution for ER Warehousing." A virtually identical Position Statement, based on a 4-hour standard, was endorsed by the Representative Forum of the Alberta Medical Association in September.

The events in BC this past Spring demonstrated that the cause of appropriate ED care is a noble one that can quickly generate a huge amount of public and media interest and support. These events also demonstrated that political mountains can be moved when large numbers of emergency physicians passionately communicate the same message. In the months and years ahead, our challenge and duty will be to advance the cause of appropriate ED care with more cohesiveness, organization, clarity of purpose, and political savvy than we have in the past. So let's stop being pushovers, and let's use the teeth for which junkyard dogs are famous. Our patients and the future of our specialty depend on it.

Competing interests: Dr. Abu-Laban was one of the majority of emergency physicians at Vancouver General Hospital who began giving selected emergency department patients a one-page statement on April 3, 2006.
Key words: emergency department overcrowding; emergency department throughput; hospital overcrowding; overcapacity protocol; full capacity protocol; admitted patients

Acknowledgement: Dr. Abu-Laban is supported by a Clincal Scholar Award from the Michael Smith Foundation for Health Research.

\section{References}

1. Fayerman P. ER 'dear patient' letters to stop. The Vancouver Sun 2006 Apr 12; Sect B:6.

2. Harris P. VGH doctors' 'Dear patient' plea 'an act of desperation.' The Vancouver Sun 2006 Apr 19;Sect B:3.

3. Yaffe B. No politician is dealing with the emergency in hospitals' ERs. The Vancouver Sun 2006 Apr 22;Sect C:7.

4. Yaffe B. Overcrowded ER killing patients at Royal Columbian, report says. Inadequate staffing is costing lives: officials. The Vancouver Sun 2006 Apr 25;Sect A:1.

5. Viccellio P. Overcrowding for dummies. ACEP News 2006; Aug:4.

6. Beveridge R, Clarke B, Janes L, et al. Canadian Emergency Department Triage and Acuity Scale: implementation guidelines. Can J Emerg Med 1999;1(3 suppl). Online version available at: www.caep.ca (accessed 2006 Oct 10).

Correspondence to: Dr. Riyad B. Abu-Laban, Research Director, Department of Emergency Medicine, Vancouver General Hospital, 855 W 12th Ave., Vancouver BC V5Z 1M9; abulaban@interchange.ubc.ca 